\title{
Research on Totem-pole Bridgeless PFC Based on Synchronous
}

\section{Reference Frame}

\author{
Weilong He ,Yongqing Wei ,Peng Zhu ,Wei Peng \\ 1. Department of Electrical Engineering, Naval University of Engineering, Wuhan 430033, China \\ 1716880658@qq.com,wyqhjgc@163.com
}

Keywords: Power factor correction; Synchronous reference frame; Current decoupling control; Stable output DC voltage

Abstract: In order to increase the control accuracy and achieve the unit power factor of totem-pole bridgeless PFC, and get sinusoidal current waveform, the method of synchronous reference frame is applied to the control of totem-pole bridgeless PFC in this paper. First of all, the working principle of totem pole bridgeless PFC is analyzed, and the topology of totem pole bridgeless PFC is improved. Secondly, the second-order generalized integrator is applied to solve the problem of the lack of an orthogonal degree of freedom in the synchronous reference frame of the totem-pole bridgeless PFC. Finally, the harmonic problem of DC side output voltage is analyzed, and the proposed algorithm is verified in Matlab/simulink simulations software platform.

\section{Introduction}

With the development of semiconductor technology, power electronics technology has been fully developed and has been deeply in all aspects of national life. Because of its superior characteristics, it is widely used in modern industry, national defense and other important fields[1]. Especially in recent years, $\mathrm{SiC}$ and $\mathrm{GaN}$ devices have appeared, which greatly reduce the loss of power electronic circuits. GaN is characterized by high frequency, high power and high temperature resistance[2]. In this paper, GaN device is applied to totem-pole bridgeless PFC. Fig. 1 shows topology of totem-pole bridgeless PFC, $S_{1}$ and $S_{2}$ of the totem-pole bridgeless are high-frequency GaN, and $S_{3}$ and $S_{4}$ are low-frequency MOSFET. Compared with common power diode, MOSFET has no problem of turning on voltage[3,4]. In Fig.2, totem-pole bridgeless PFC reduces the number of semiconductors compared to conventional single-phase rectifiers. To improve the power factor $[5,6,7]$, the totem-pole bridgeless PFC must control the system current of the electrified wire netting. The phase difference is 0 between the system current of the electrified wire netting and the system voltage of the electrified wire netting, thus ensuring the work of the totem-pole bridgeless PFC to work in the unit power factor[8]. In the current common control methods, the current loop control is basically PI regulation, which in DC will achieve no difference regulation[9], but AC signal can't be achieved. In this paper, the control method of synchronous reference frame is put forward for the regulation of system current of the electrified wire netting, which can change the input sinusoidal current into two virtual direct signals through synchronous reference frame. And then two virtual direct signals are adjusted by PI regulation, and which will turn into high quality sine wave. Synchronous reference frame requires two signals, but there is only one current signal for totem-pole bridgeless PFC, so we must construct two orthogonal signals. In this paper, we use the second-order generalized integrator to construct two orthogonal signals. 


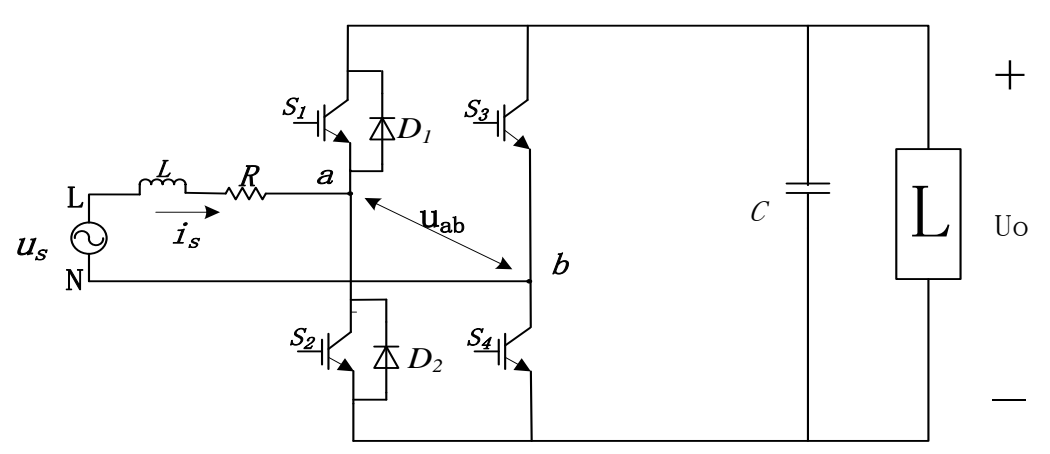

Figure 1 Totem-pole PFC topology

\section{Principle analysis of totem pole bridgeless PFC}

In Fig.1, the totem-pole bridgeless PFC has two high-frequency GaN and two common MOSFET. The two low-frequency MOSFET $S_{3}$ and $S_{4}$ are alternately turned on along with the positive and negative half cycles of the power supply. In Fig.2, when the input voltage is in the positive half - cycle and $\mathrm{S}_{4}$ is driven, the circuit is divided into two modes of operation. In Fig.2. (a), when $S_{2}$ is turned on, the current path is the end of the power supply L-inductor $L-S_{2}-S_{4}$ - power supply N.At this moment the power supply charges the inductor, and the energy storage of the inductor is increased, and the load is powered by an output capacitor. In Fig.2. (b), when $\mathrm{S}_{2}$ is disconnected, the current path is power supply L-inductor L- $\mathrm{D}_{1}$-load $-\mathrm{S}_{4}$-power supply N. Power supply and inductor supply power to load, and the energy storage of inductor is reduced. The same working in the negative half of the week.

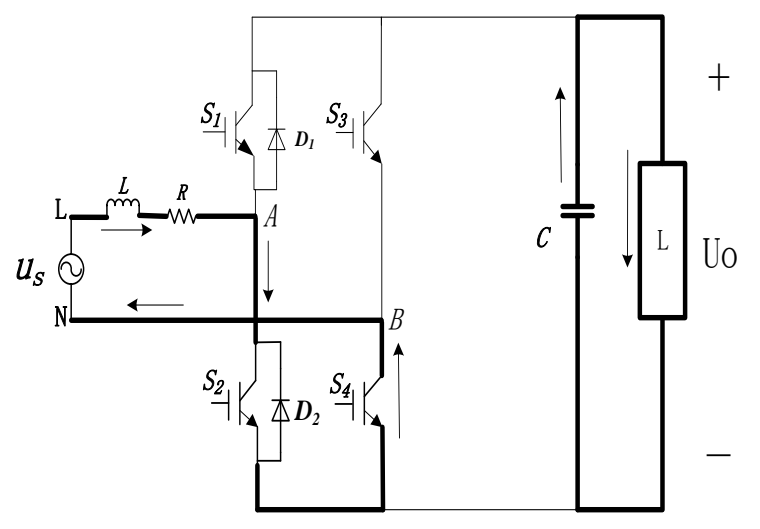

(a) Mode 1

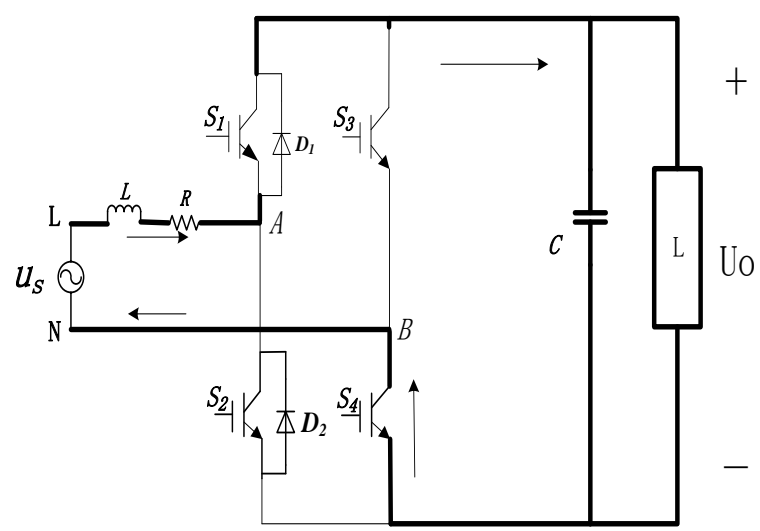

(b) Mode 2

Figure 2 Positive half-cycle working mode of totem pole bridgeless PFC

According to the topology analysis of totem-pole bridgeless PFC, only two semiconductor devices are in the working state in a switching period, the on-state loss is small and the CM EMI equivalent model is small. In the totem-pole bridgeless PFC, the body diodes $\mathrm{D}_{1}$ and $\mathrm{D}_{2}$ of the switching tubes $S_{1}$ and $S_{2}$ have longer recovery time compared with the conventional diodes. And the reverse recovery loss is serious if the switching tubes $S_{1}$ and $S_{2}$ are operated in the current continuous mode, so the totem-pole bridgeless PFC generally operates in discontinuous current mode (DCM) or between current mode (BCM). Although totem-pole bridgeless PFC has the disadvantages, the advantages are more prominent. Therefore, the bridgeless PFC circuit has a good application prospect in medium and small power occasions. 


\section{Controlling principle analysis of totem pole bridgeless PFC}

3.1 Principle and decoupling control of single - phase synchronous reference frame. The current control of totem-pole bridgeless PFC is the core of power factor control. In this paper, the current inner loop control based on synchronous reference frame, which is proposed to realize the current regulation without difference. Specifically, the AC signal is changed into virtual DC signal in current loop by synchronous reference frame, and then virtual DC signal is adjusted by PI, and then changed into sinusoidal current signal by synchronous reference frame. In Fig.1, L is input inductance and $\mathrm{R}$ is input side equivalent resistance, $\mathrm{u}_{a b}$ is input voltage. The $u_{s}$ voltage equation can be obtained by KVL.

$$
u_{s}=L \frac{d i_{s}}{d t}+R i_{s}+u_{a b}
$$

The equation (1) is used in both the $\alpha-\beta$ static reference frame and the $d-q$ synchronous reference frame, where $\mathrm{R}$ is the input resistance on line side, and generally $\mathrm{R}$ is relatively small. So it can be ignored, after getting the vector equation.

$$
\boldsymbol{u}_{\boldsymbol{s}}=L \frac{d \boldsymbol{i}_{\boldsymbol{s}}}{d t}+\boldsymbol{u}_{\boldsymbol{a b}}
$$

When the starting position of the d-q synchronous reference frame is same with the $\alpha-\beta$ static reference frame, the transformation matrix between the two coordinate systems is.

$$
\left[\begin{array}{l}
d \\
q
\end{array}\right]=\left[\begin{array}{cc}
\sin \omega t & \cos \omega t \\
-\cos \omega t & \sin \omega t
\end{array}\right]\left[\begin{array}{l}
\alpha \\
\beta
\end{array}\right] \text {. }
$$

The voltage signals of the voltage vector $\mathrm{u}_{s}$ on line side are $\mathrm{u}_{s \alpha}$ and $\mathrm{u}_{s \beta}$ in the $\alpha-\beta$ static reference frame, and the signals of the current vector $i_{s}$ are $i_{s \alpha}$ and $i_{s \beta}$.

$$
\begin{gathered}
\left\{\begin{array}{c}
\mathrm{u}_{s \alpha}=u_{s}=U_{m} \sin \omega t \\
\mathrm{u}_{s \beta}=U_{m} \cos \omega t
\end{array}\right. \\
\left\{\begin{array}{c}
\mathrm{i}_{s \alpha}=i_{s}=I_{m} \sin \omega t \\
\mathrm{i}_{s \beta}=I_{m} \cos \omega t
\end{array} .\right.
\end{gathered}
$$

$U_{m}, I_{m}$ are the peak values of voltage and current on line side.

By substituting equation ( 3 ), ( 4 ) and ( 5 ) into equation ( 2 ), the voltage components $\mathrm{u}_{s d}$ and $\mathrm{u}_{\mathrm{s} q}$ of voltage on line side in the d-q synchronous reference frame can be simplified as follows.

$$
\left\{\begin{array}{l}
\mathrm{u}_{s d}=L \frac{d i_{s d}}{d t}-\omega L i_{s q}+u_{a b d} \\
\mathrm{u}_{s q}=L \frac{d i_{s q}}{d t}+\omega L i_{s d}+u_{a b q} .
\end{array}\right.
$$

After transformation, $u_{a b d}$ and $u_{a b q}$ are the components of $\mathrm{u}_{a b}$ in $\mathrm{d}-\mathrm{q}$ synchronous reference frame.

$$
\left\{\begin{array}{l}
u_{a b d}=\mathrm{u}_{s d}-L \frac{d i_{s d}}{d t}+\omega L i_{s q} \\
u_{a b q}=\mathrm{u}_{s q}-L \frac{d i_{s q}}{d t}-\omega L i_{s d}
\end{array}\right.
$$

From equation (7), through synchronous reference frame, there is cross coupling between current 
in d-q synchronous reference frame, introducing decoupling control in the control system. As shown is decoupling control block diagram in Fig.3, eliminating the system error caused by coupling.

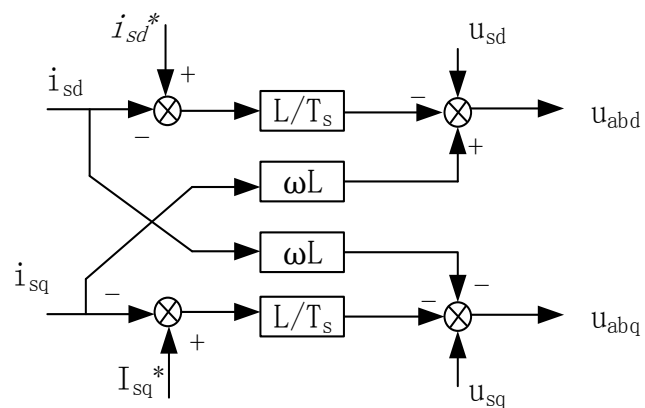

Figure 3 The decoupling control principle

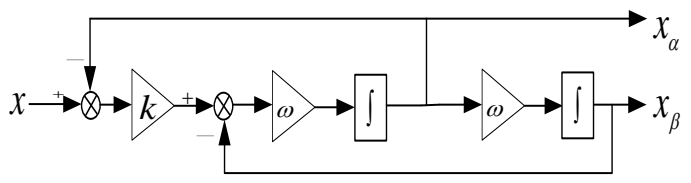

Figure 4 The second-order generalized integrator

3.2 Construction of single-phase virtual orthogonal current. In the three-phase system, the dq current control is transformed through two coordinate transformations. First of all, the three-phase $\mathrm{AC}$ is converted into two phase $\mathrm{AC}$ under the $\alpha-\beta$ static reference frame by Clarke transformation. Secondly, the two phases $\alpha-\beta$ static reference frame is transformed into two phase DC in the $d-q$ synchronous reference frame. But in single phase system, there is a degree of freedom in synchronous reference frame, so it is impossible to coordinate transformation directly. Two orthogonal components are constructed before the rotational coordinate transformation. In this paper, the orthogonal current components are obtained by the second-order generalized integrator. As shown in Fig.3 is the second-order generalized integrator structure block diagram, and $\mathrm{x}_{\alpha}$ and $\mathrm{x}_{\beta}$ are original input signals and orthogonal input signals, respectively.

The transfer function of the second-order generalized integrator is shown in Fig. 4.

$$
\left\{\begin{array}{l}
L\left(\frac{x_{\alpha}}{x}\right)=\frac{k \omega s}{s^{2}+k \omega s+\omega^{2}} \\
L\left(\frac{x_{\alpha \beta}}{x}\right)=\frac{k \omega^{2}}{s^{2}+k \omega s+\omega^{2}} .
\end{array}\right.
$$

In equation (8) $\mathrm{k}$ is the damping coefficient and $\omega$ is the fundamental frequency of the grid voltage. From the transfer function of the second-order generalized integrator, we can get that the essence of the second-order generalized integrator, which are an orthogonal pair composed of a second-order band-pass filter and a second-order low-pass filter, whose characteristics are determined by the size of damping coefficient $\mathrm{k}$. The larger the $\mathrm{k}$ is, the better the filtering performance of the system's input is, and the better the steady-state performance is. The smaller the $\mathrm{k}$ is, the more sensitive the system is, and the dynamic performance is good.

3.3 Harmonic analysis of totem-pole bridgeless PFC. The output DC voltage of totem-pole bridgeless PFC has the inherent harmonic of double-frequency of voltage on line side. Assuming that totem-pole bridgeless PFC works in ideal state, the input voltage on line side is $u_{s}$ and the current on line side is $i_{s}$.

$$
u_{s}=U_{m} \sin \omega t
$$




$$
i_{s}=I_{m} \sin (\omega t-\theta) \text {. }
$$

Where $U_{m}$ is the peak values of voltage on line side and $I_{m}$ is the peak values of current on line side.

According to equation (9) and equation (10), the instantaneous power of the totem-pole bridgeless PFC on line side can be obtained. Totem-pole bridgeless PFC online side instantaneous power is $P_{i}$.

$$
P_{i}=U_{m} \sin (\omega t) \cdot I_{m} \sin (\omega t-\theta)=\frac{U_{m} I_{m}}{2}[\cos (\theta)-\cos (2 \omega t-\theta)] .
$$

Totem-pole bridgeless PFC DC side instantaneous power is $P_{o} . \theta$ is the phase difference between voltage and current on line side, when $\theta$ is equal to 0 , the power factor is 1 .

$$
P_{o}=U_{d c} \cdot I_{d c}+C u_{d c} \frac{d \bar{u}_{d c}}{d t} \text {. }
$$

As shown in the equation (12), $U_{d c}$ is the average output voltage and $I_{d c}$ is the average output current. $\bar{u}_{d c}$ is ripple voltage on the DC side and $C$ is the DC side support capacitor.

Under ideal conditions, the input instantaneous power $P_{i}$ and the output instantaneous power $P_{o}$ are equal. We can be obtained equation (13) and equation (14), comparing equation (11) with equation (12).

$$
\begin{aligned}
& C u_{d c} \frac{d \bar{u}_{d c}}{d t}=-\frac{U_{m} I_{m}}{2} \cos (2 \omega t-\theta) . \\
& U_{d c} \cdot I_{d c}=\frac{U_{m} I_{m}}{2} \cos \theta .
\end{aligned}
$$

From equation (13), We can obtain $\bar{u}_{d c}$.

$$
\bar{u}_{d c}=-\frac{U_{m} I_{m}}{4 \omega C u_{d c}} \cos (2 \omega t-\theta) .
$$

From equation (14), it is possible to obtain harmonics of the voltage on the grid side containing the double frequency in the DC bus voltage.

\section{System simulation analysis}

In order to verify the correctness and effectiveness of the control method of the totem pole bridgeless PFC based on synchronous reference frame, a simulation model is built in the MATLAB / Simulink simulation software platform. Simulation result is shown in Fig. 5. Through the analysis, the sine of the current on line side of the totem-pole bridgeless PFC based on synchronous reference frame is better, and the phase difference $\theta$ between the voltage on line side $u_{s}$ and the current on line side $i_{s}$ can be ensured to be approximately equal to 0 .

In Fig.5, it can be seen that two orthogonal current signals $\mathrm{i}_{s \alpha}$ and $\mathrm{i}_{s \beta}$ can be obtained by the second-order generalized integrator. In Fig.6, it can be seen the voltage $U_{d c}$ output from the DC side has harmonics, and the harmonic frequency of the voltage output from the DC side is twice the voltage frequency of the grid side. 

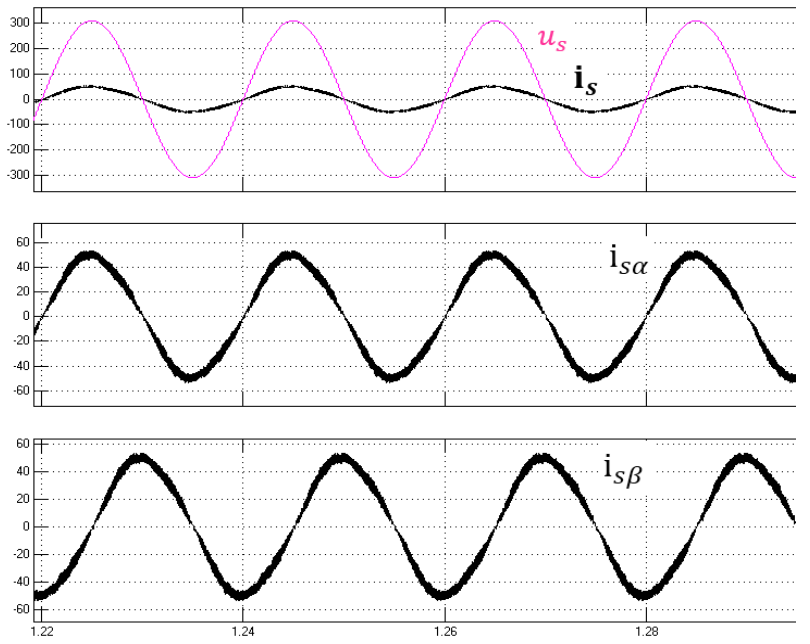

Figure 5 Voltage and current on line side base

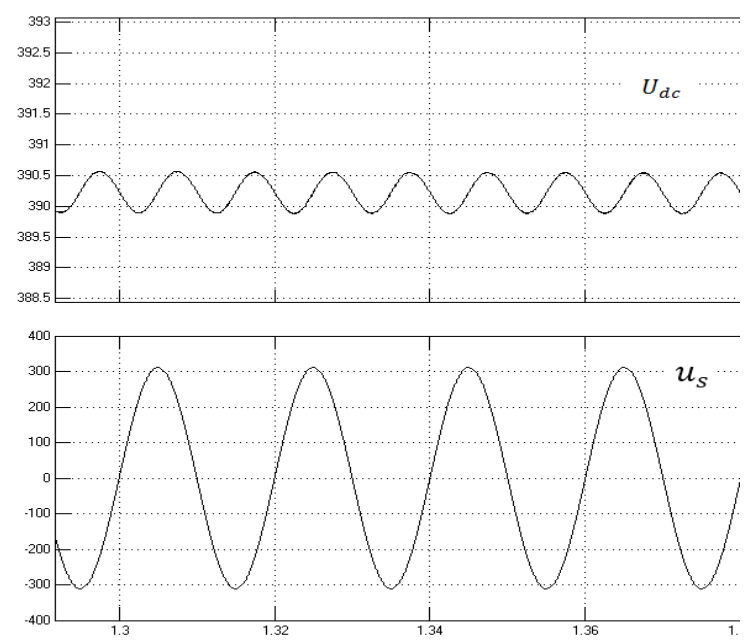

Figure 6 Output DC voltage

\section{Conclusions and prospect}

In this paper, the method of synchronous reference frame is applied to the control of totem-pole bridgeless PFC, and the result of simulation shows that the harmonic of current on line side is small, and the phase difference $\theta$ between $u_{s}$ and $i_{s}$ is equal to 0 , so that the totem-pole bridge PFC operates at unity power factor, while the output DC side voltage is stable.

The second harmonic problem of the output DC voltage may not be eliminated when the external voltage loop is controlled, so the adjustment of the inner loop of the current will be affected, which will be studied in the future.

\section{References}

[1] Ma W, Fei X, Nie S. Applications and Development of Power Electronics in Electromagnetic Launch System[J]. Transactions of China Electrotechnical Society, 2016.

[2] Rong Chuicai. Research on the Model of Ga N HEMT Device and High Efficiency Power Amplifier [D]. Research on the Model of Ga N HEMT Device and High Efficiency Power Amplifier, 2017.

[3] Jang Y, Jovanovic M M. A Bridgeless PFC Boost Rectifier With Optimized Magnetic Utilization[J]. IEEE Transactions on Power Electronics, 2009, 24(1):85-93.

[4] Choi W Y, Kwon J M, Kwon B H. Bridgeless dual-boost rectifier with reduced diode reverserecovery problems for power-factor correction[J]. Iet Power Electronics, 2008, 1(2):194-202.

[5] Wang Y. An Interleaved Totem-Pole Bridgeless Boost PFC Rectifier with Zero-Ripple Current Filter[J]. Transactions of China Electrotechnical Society, 2011, 26(9):175-182

[6] Yang J J, Wang L W, Wang S Q, et al. A High Efficiency Bridgeless PFC Circuit based on One Cycle Control Strategy[J]. Power Electronics, 2009, 43(7):13-14.

[7] Wei H U, Kang Y, Hao H U, et al. Semi Bridgeless Boost PFC Converter With Quasi-zero-current Switching Auxiliary Switch[J]. Proceedings of the Csee, 2017.

[8] Hao L, Lin M, Yang C, et al. Forced Excitation Control of Doubly-fed Wind Power Systems During Low Voltage Ride-through Based on Sine Wave Shaped Stator Current[J]. Automation of Electric Power Systems, 2014, 38(18):20-26.

[9] Chen Z, Luo Y, Zhu Y, et al. Analysis and Design of Three-phase Rectifier With Near-sinusoidal Input Currents[J]. Proceedings of the Csee, 2009, 29(36):1703-1 - 1703-7. 